\title{
On the Nonlinear Fractional Differential Equations with Caputo Sequential Fractional Derivative
}

\author{
Hailong $\mathrm{Ye}^{1}$ and Rui Huang ${ }^{1,2}$ \\ ${ }^{1}$ School of Mathematical Sciences, South China Normal University, Guangzhou 510631, China \\ ${ }^{2}$ School of Mathematics, South China University of Technology, Guangzhou 510641, China \\ Correspondence should be addressed to Rui Huang; huangrui@m.scnu.edu.cn
}

Received 3 August 2015; Accepted 27 October 2015

Academic Editor: Ivan Avramidi

Copyright (c) $2015 \mathrm{H}$. Ye and R. Huang. This is an open access article distributed under the Creative Commons Attribution License, which permits unrestricted use, distribution, and reproduction in any medium, provided the original work is properly cited.

\begin{abstract}
The purpose of this paper is to investigate the existence of solutions to the following initial value problem for nonlinear fractional differential equation involving Caputo sequential fractional derivative ${ }^{c} D_{0}^{\alpha_{2}}\left(\left|{ }^{c} D_{0}^{\alpha_{1}} y(x)\right|^{p-2 c} D_{0}^{\alpha_{1}} y(x)\right)=f(x, y(x)), x>0, y(0)=b_{0}$, ${ }^{c} D_{0}^{\alpha_{1}} y(0)=b_{1}$, where ${ }^{c} D_{0}^{\alpha_{1}},{ }^{c} D_{0}^{\alpha_{2}}$ are Caputo fractional derivatives, $0<\alpha_{1}, \alpha_{2} \leq 1, p>1$, and $b_{0}, b_{1} \in \mathbb{R}$. Local existence of solutions is established by employing Schauder fixed point theorem. Then a growth condition imposed to $f$ guarantees not only the global existence of solutions on the interval $[0,+\infty)$, but also the fact that the intervals of existence of solutions with any fixed initial value can be extended to $[0,+\infty)$. Three illustrative examples are also presented. Existence results for initial value problems of ordinary differential equations with $p$-Laplacian on the half-axis follow as a special case of our results.
\end{abstract}

\section{Introduction}

This paper deals with the following initial value problem for nonlinear fractional differential equation involving Caputo sequential fractional derivative:

$$
\begin{aligned}
{ }^{c} D_{0}^{\alpha_{2}}\left(\left|{ }^{c} D_{0}^{\alpha_{1}} y(x)\right|^{p-2}{ }^{c} D_{0}^{\alpha_{1}} y(x)\right) & =f(x, y(x)), \\
x>0, & \\
y(0) & =b_{0}, \\
{ }^{c} D_{0}^{\alpha_{1}} y(0) & =b_{1},
\end{aligned}
$$

where ${ }^{c} D_{0}^{\alpha_{1}},{ }^{c} D_{0}^{\alpha_{2}}$ are Caputo fractional derivatives, $0<\alpha_{1}$, $\alpha_{2} \leq 1, p>1$, and $b_{0}, b_{1} \in \mathbb{R}$, and $x^{\sigma} f(x, y)$ is continuous on $[0,+\infty) \times \mathbb{R}, 0 \leq \sigma<\alpha_{2}$. When $p=2$, the equation in (1) becomes a sequential fractional differential equation. Here, we follow the definition of sequential fractional derivative presented by Podlubny [1]:

$$
\mathscr{D}^{v} y(x)=D^{\nu_{1}} D^{\nu_{2}} \cdots D^{\nu_{m}} y(x), \quad m \in \mathbb{N}_{+} \text {, }
$$

where the symbol $D^{\gamma_{i}}(i=1,2, \ldots, m)$ means the RiemannLiouville derivative or the Caputo derivative. It is easy to see that (2) is a generalized expression presented by Miller and Ross in [2].

Fractional differential equations have been of great interest for the past three decades; see the monographs [14] and the papers of [5-8]. This is due to the intensive development of the theory of fractional calculus itself as well as its applications. Apart from diverse areas of pure mathematics, fractional differential equations can be used in modeling of various fields of science and engineering such as rheology, self-similar dynamical processes, porous media, fluid flows, viscoelasticity, electrochemistry, control, electromagnetic, and many other branches of science. For details, see [9-12] and the references therein.

Recently, we note that the investigation for the existence of solutions of sequential fractional differential equations associated with a variety of initial and boundary value conditions has attracted the considerable attention of researchers. Here, we mention some works on them. In [13, 14], the authors investigated a class of Riemann-Liouville sequential fractional differential equation

$$
\left(D_{0}^{\alpha} y\right)^{\prime}+a(x) y=0, \quad x \geq 0,
$$

and obtained existence, nonexistence, and asymptotic property of the solutions by some nonlinear analysis methods. In 
$[15,16]$, the authors considered the existence and uniqueness of the solutions for initial value problems involving RiemannLiouville sequential fractional derivative by using monotone iterative method and fixed point method. As for sequential fractional differential equations associated with boundary value conditions, we refer the reader to [17-22]. For example, Chen and Tang [21] considered the existence of the solutions for the following Caputo sequential fractional differential equation:

$$
\begin{aligned}
\left(a(t){ }^{c} D_{0+}^{\alpha} u(t)\right)^{\prime}=f\left(t, u(t), u^{\prime}(t),{ }^{c} D_{0+}^{\alpha} u(t)\right) & \\
& t \in[0,1],
\end{aligned}
$$

with $m$-point boundary value conditions by the coincidence degree continuation theorem. After that, in 2015, Jiang [22] investigated the following Riemann-Liouville sequential fractional differential equations with $p$-Laplacian at resonance:

$$
\begin{aligned}
& D_{0+}^{\beta}\left(\varphi_{p}\left(D_{0+}^{\alpha} u\right)\right)(t)+f\left(t, u(t), D_{0+}^{\alpha-1} u(t), D_{0+}^{\alpha} u(t)\right) \\
& \quad=0, \quad t \in[0,1]
\end{aligned}
$$

where $\varphi_{p}(s)=|s|^{p-2} s, p>1$. By the extension of the continuous theorem in [23] and constructing suitable operators, they obtained the existence of solutions satisfied integral boundary value conditions.

In view of the facts that the Laplace transform of the Caputo derivative allows utilization of initial values of classical integer-order derivatives and that the Caputo derivative of a constant is 0 , sequential fractional differential equations involving the Caputo fractional derivative have more clear physical interpretations than those involving the RiemannLiouville fractional derivative (see $[3,4]$ ).

To the best of our knowledge, there is no paper dealing with the existence of solutions of Caputo sequential fractional differential equations with initial value conditions. In our latest paper [24], by virtue of uniform Lipschitz continuity of $x^{\sigma} f(x, \cdot)$ on $\mathbb{R}$ for every $x>0$, we proved the existence and uniqueness of solutions of problem (1). Now, in this paper, we are concerned with the initial value problem (1) without uniform Lipschitz continuity of $x^{\sigma} f(x, \cdot)$. By fractional Taylor expansion theorem, we first obtain an integral equation equivalent to the initial value problem (1), to which local existence of solutions is established utilizing Schauder fixed point theorem. Then a growth condition imposed to $f$ guarantees not only the global existence of solutions on the interval $[0,+\infty)$, but also the fact that the intervals of existence of solutions with any fixed initial value can be extended to $[0,+\infty)$. In addition, existence results for initial value problems of ordinary differential equations with $p$ Laplacian on the half-axis follow as a special case of our results.

The paper is organized as follows. In Section 2, we present some necessary definitions and preliminary results that will be used in our discussions. The main results and their proofs are given in Section 3. In Section 4, we will give three examples to illustrate our results.

\section{Preliminaries}

In this section, we introduce some basic definitions and notations (see the monographs [1,2] for further details) and give several useful preliminary results which are used throughout this paper.

Definition 1. Let $\alpha>0$. The Riemann-Liouville fractional integral of a function $y:(0,+\infty) \rightarrow \mathbb{R}$ of order $\alpha$ is given by

$$
J_{0}^{\alpha} y(x):=\frac{1}{\Gamma(\alpha)} \int_{0}^{x}(x-t)^{\alpha-1} y(t) d t
$$

provided that the right-hand side is pointwise defined on $(0,+\infty)$. Here and in what follows $\Gamma$ is the Gamma function.

The fractional integration operator $J_{a}^{\alpha}$ has that the following semigroup property

$$
J_{0}^{\alpha} J_{0}^{\beta} y(x)=J_{0}^{\beta} J_{0}^{\alpha} y(x)=J_{0}^{\alpha+\beta} y(x)
$$

holds for all $\alpha>0, \beta>0$.

Definition 2. Let $\alpha>0$ and let $n$ be the smallest integer that exceeds $\alpha$. The Riemann-Liouville fractional derivative of a continuous function $y:(0,+\infty) \rightarrow \mathbb{R}$ of order $\alpha$ is given by

$$
D_{0}^{\alpha} y(x):=\frac{1}{\Gamma(n-\alpha)}\left(\frac{d}{d x}\right)^{n} \int_{0}^{x}(x-t)^{n-\alpha-1} y(t) d t
$$

provided that the right-hand side is pointwise defined on $(0,+\infty)$.

Obviously, the Riemann-Liouville fractional differentiation is the left inverse of the Riemann-Liouville fractional integration for continuous function $y$ in the following sense:

$$
D_{0}^{\alpha} J_{0}^{\alpha} y(x)=y(x)
$$

for $\alpha>0$. However, it is not the right inverse. More precisely, we have the following fractional Taylor expansion theorem.

Theorem 3. Let $0<\alpha<1$. Assume that $y$ is such that $J_{0}^{1-\alpha} y$ is absolutely continuous. Then

$$
J_{0}^{\alpha} D_{0}^{\alpha} y(x)=y(x)-\frac{x^{\alpha-1}}{\Gamma(\alpha)} \lim _{z \rightarrow 0+} J_{0}^{1-\alpha} y(z), \quad x \geq 0 .
$$

Definition 4. Let $\alpha>0$ and let $n$ be the smallest integer that exceeds $\alpha$. The Caputo fractional derivative of a continuous function $y:(0,+\infty) \rightarrow \mathbb{R}$ of order $\alpha$ is given by

$$
{ }^{c} D_{0}^{\alpha} y(x):=\left(D_{0}^{\alpha}\left[y-\sum_{k=0}^{n-1} \frac{y^{(k)}(0)}{k !}(\cdot)^{k}\right]\right)(x)
$$

provided that the right-hand side is pointwise defined on $(0,+\infty)$.

The definition of solutions of the initial value problem (1) is given as follows. 
Definition 5. Let $T>0$; a function $y$ is called a solution of (1) on $[0, T]$, if

(i) $y,{ }^{c} D^{\alpha_{1}} y \in C([0, T]),{ }^{c} D_{0}^{\alpha_{2}}\left(\left|{ }^{c} D_{0}^{\alpha_{1}} y\right|^{p-2 c} D_{0}^{\alpha_{1}} y\right) \in C((0$, $T])$;

(ii) $y$ satisfies problem (1) on $(0, T]$.

Definition 6. A function $y$ is called a solution of (1) on $[0,+\infty)$, if for any $T>0, y$ is a solution of problem (1) on $[0, T]$.

In order to study the existence of solutions of (1), we should transform problem (1) into an equivalent integral equation. We need the following two lemmas.

Lemma 7. Let $\alpha, \mu>0$; then one has

$$
\int_{a}^{b}(b-t)^{\alpha-1}(t-a)^{\mu-1} d t=\frac{\Gamma(\alpha) \Gamma(\mu)}{\Gamma(\alpha+\mu)}(b-a)^{\alpha+\mu-1} .
$$

Lemma 8. Let $0<\mu<\alpha \leq 1$. If $g$ is a continuous function defined on $[0,+\infty)$, then $\int_{0}^{x}(x-t)^{\alpha-1} t^{-\mu} g(t) d t$ is continuous with respect to $x$ in $[0,+\infty)$.

Proof. Let $x_{0}>0$ and take $\delta_{0} \in\left(0, x_{0}\right)$. For $|\delta|<\delta_{0}$, we have

$$
\begin{aligned}
& \mid \int_{0}^{x_{0}+\delta}\left(x_{0}+\delta-t\right)^{\alpha-1} t^{-\mu} g(t) d t-\int_{0}^{x_{0}}\left(x_{0}-t\right)^{\alpha-1} \\
& \cdot t^{-\mu} g(t) d t|\leq| \int_{0}^{x_{0}+\delta}\left(x_{0}+\delta-t\right)^{\alpha-1} t^{-\mu} g(t) d t \\
& \quad-\int_{0}^{x_{0}}\left(x_{0}+\delta-t\right)^{\alpha-1} t^{-\mu} g(t) d t \mid \\
& \quad+\mid \int_{0}^{x_{0}}\left(x_{0}+\delta-t\right)^{\alpha-1} t^{-\mu} g(t) d t-\int_{0}^{x_{0}}\left(x_{0}-t\right)^{\alpha-1} \\
& \quad \cdot t^{-\mu} g(t) d t|=| \int_{x_{0}}^{x_{0}+\delta}\left(x_{0}+\delta-t\right)^{\alpha-1} t^{-\mu} g(t) d t \mid \\
& \quad+\int_{0}^{x_{0}} \mid\left(\left(x_{0}+\delta-t\right)^{\alpha-1}-\left(x_{0}-t\right)^{\alpha-1}\right) \\
& \quad \cdot t^{-\mu} g(t) \mid d t .
\end{aligned}
$$

Since $g$ is continuous and bounded in the neighborhood of $x_{0}$, we conclude that

$$
\begin{gathered}
\left|\int_{x_{0}}^{x_{0}+\delta}\left(x_{0}+\delta-t\right)^{\alpha-1} t^{-\mu} g(t) d t\right| \leq C\left(x_{0}+\delta\right)^{\alpha-1} \\
\cdot\left|\int_{x_{0}}^{x_{0}+\delta}\left(1-\frac{t}{x_{0}+\delta}\right)^{\alpha-1} t^{-\mu} d t\right| \leq C\left(x_{0}+\delta\right)^{\alpha-\mu} \\
\cdot\left|\int_{x_{0} /\left(x_{0}+\delta\right)}^{1}(1-z)^{\alpha-1} z^{-\mu} d z\right| \leq C \frac{\delta^{\alpha}}{\alpha x_{0}^{\mu}}
\end{gathered}
$$

$$
\begin{aligned}
& \int_{0}^{x_{0}}\left|\left(\left(x_{0}+\delta-t\right)^{\alpha-1}-\left(x_{0}-t\right)^{\alpha-1}\right) t^{-\mu} g(t)\right| d t \\
& \quad \leq C x_{0}^{\alpha-\mu} \int_{0}^{1}\left|\left(\frac{x_{0}+\delta}{x_{0}}-z\right)^{\alpha-1}-(1-z)^{\alpha-1}\right| z^{-\mu} d z \\
& \quad=C x_{0}^{\alpha-\mu} \mid \int_{0}^{1}(1-z)^{\alpha-1} z^{-\mu} d z \\
& \quad-\int_{0}^{1+\delta / x_{0}}\left(1+\frac{\delta}{x_{0}}-z\right)^{\alpha-1} z^{-\mu} d z \\
& +\int_{1}^{1+\delta / x_{0}}\left(1+\frac{\delta}{x_{0}}-z\right)^{\alpha-1} z^{-\mu} d z \mid \\
& \quad \leq C x_{0}^{\alpha-\mu} \mid \int_{0}^{1}(1-z)^{\alpha-1} z^{-\mu} d z \\
& \quad-\int_{0}^{1+\delta / x_{0}}\left(1+\frac{\delta}{x_{0}}-z\right)^{\alpha-1} z^{-\mu} d z \mid \\
& +C x_{0}^{\alpha-\mu}\left|\int_{1}^{1+\delta / x_{0}}\left(1+\frac{\delta}{x_{0}}-z\right)^{\alpha-1}(z-1)^{-\mu} d z\right| \\
& \quad \leq C x_{0}^{\alpha-\mu} \frac{(\alpha) \Gamma(1-\mu)}{\Gamma(\alpha+1-\mu)} \mid 1-\left(1+\frac{\delta}{x_{0}}\right)^{\alpha-\mu} \\
& +\left(\frac{\delta}{x_{0}}\right)^{\alpha-\mu} \mid,
\end{aligned}
$$

where $C=\sup _{0 \leq x \leq x_{0}+\delta_{0}}|g(x)|$. Combining (13), (14) with (15), we arrive at

$$
\begin{gathered}
\lim _{\delta \rightarrow 0} \mid \int_{0}^{x_{0}+\delta}\left(x_{0}+\delta-t\right)^{\alpha-1} t^{-\mu} g(t) d t \\
-\int_{0}^{x_{0}}\left(x_{0}-t\right)^{\alpha-1} t^{-\mu} g(t) d t \mid=0 .
\end{gathered}
$$

In addition, it is easy to see that

$$
\left|\int_{0}^{x} x^{\alpha-1} t^{-\mu} g(t) d t\right| \leq C x^{\alpha-\mu} .
$$

Therefore, $\int_{0}^{x}(x-t)^{\alpha-1} t^{-\mu} g(t) d t$ is continuous with respect to $x$ in $[0,+\infty)$.

Remark 9. If $y \in C([0,+\infty))$, then $x^{\sigma} f(x, y(x))$ is continuous on $[0,+\infty)$. According to Lemma 8 , the function

$$
J_{0}^{\alpha_{2}} f(x, y(x))=\frac{1}{\Gamma\left(\alpha_{2}\right)} \int_{0}^{x}(x-t)^{\alpha_{2}-1} f(t, y(t)) d t
$$

is also continuous in $[0,+\infty)$ and $J_{0}^{\alpha_{2}} f(0, y(0))=0$.

Now we are ready to transform problem (1) into an equivalent integral equation. For the reader's convenience, we list two special notations that will be used in the following paper: $q=p /(p-1)$ and $\phi_{q}(s)=|s|^{q-2} s$ for $s \in \mathbb{R}$. 
Proposition 10. A function y defined in $C([0, T])$ is a solution of problem (1) if and only if it satisfies the following integral equation on $[0, T]$ :

$$
y(x)=b_{0}+\frac{1}{\Gamma\left(\alpha_{1}\right)} \int_{0}^{x}(x-t)^{\alpha_{1}-1} \Phi_{y}(t) d t,
$$

where

$$
\begin{aligned}
& \Phi_{y}(x)=\phi_{q}\left(\phi_{p}\left(b_{1}\right)\right. \\
& \left.+\frac{1}{\Gamma\left(\alpha_{2}\right)} \int_{0}^{x}(x-t)^{\alpha_{2}-1} f(t, y(t)) d t\right) .
\end{aligned}
$$

Proof. First we prove the necessity. Let $y \in C([0, T])$ be a solution of problem (1) and define

$$
g(x)=\left|{ }^{c} D_{0}^{\alpha_{1}} y(x)\right|^{p^{-2} c} D_{0}^{\alpha_{1}} y(x),
$$

and then $g \in C([0, T])$ and $g(0)=\phi_{p}\left(b_{1}\right)$. According to Definition 4 and Theorem 3, the differential equation of problem (1) can be transformed into the following form:

$$
f(x, y(x))=D J_{0}^{1-\alpha_{2}}(g-g(0))(x) .
$$

Obviously, $J_{0}^{1-\alpha_{2}}(g-g(0))$ is absolutely continuous on $[0, T]$. Combining with Theorem 3 , we have

$$
\begin{aligned}
g(x) & =g(0)+J_{0}^{\alpha_{2} c} D_{0}^{\alpha_{2}} g(x) \\
& =\phi_{p}\left(b_{1}\right)+J_{0}^{\alpha_{2}} f(\cdot, y(\cdot))(x) .
\end{aligned}
$$

That is,

$$
{ }^{c} D_{0}^{\alpha_{1}} y(x)=\phi_{q}\left(\phi_{p}\left(b_{1}\right)+J_{0}^{\alpha_{2}} f(\cdot, y(\cdot))(x)\right) .
$$

Applying Definition 4 and Theorem 3 again, we have

$$
{ }^{c} D_{0}^{\alpha_{1}} y(x)=D J_{0}^{1-\alpha_{1}}(y-y(0))(x) .
$$

Obviously, $J_{0}^{1-\alpha_{1}}(y-y(0)) \in C^{1}([0, T])$. Combining with Theorem 3, we have

$$
\begin{aligned}
y(x) & =y(0)+J_{0}^{\alpha_{1}} D_{0}^{\alpha_{1}}(y-y(0))(x) \\
& =y(0)+J_{0}^{\alpha_{1} c} D_{0}^{\alpha_{1}} y(x)=b_{0}+J_{0}^{\alpha_{1}} \Phi_{y}(x) .
\end{aligned}
$$

Therefore, $y$ satisfies the integral equation (19).

Next, we prove the sufficiency. Let $y \in C([0, T])$ be a solution of the integral equation (19). Combining with Definition 1, (19) reduces to

$$
y(x)=b_{0}+J_{0}^{\alpha_{1}} \Phi_{y}(x) .
$$

From Remark 9, we see that $J_{0}^{\alpha_{1}} \Phi_{y} \in C([0, T])$ and $J_{0}^{\alpha_{1}} \Phi_{y}(0)=0$. That is, $y \in C([0, T])$ and $y(0)=b_{0}$. Applying the operator ${ }^{c} D^{\alpha_{1}}$ to both sides of (27), we obtain that

$$
\begin{aligned}
{ }^{c} D_{0}^{\alpha} y(x) & ={ }^{c} D_{0}^{\alpha}\left(b_{0}+J_{0}^{\alpha_{1}} \Phi_{y}(x)\right)=D_{0}^{\alpha} J_{0}^{\alpha_{1}} \Phi_{y}(x) \\
& =\Phi_{y}(x) .
\end{aligned}
$$

Then we have ${ }^{c} D_{0}^{\alpha} y \in C([0, T])$ and ${ }^{c} D_{0}^{\alpha} y(0)=\Phi_{y}(0)=b_{1}$. By virtue of $g$, we transform the above equation into the following form:

$$
g(x)=\phi_{p}\left(b_{1}\right)+J_{0}^{\alpha_{2}} f(\cdot, y(\cdot))(x) .
$$

Similarly, applying the operator ${ }^{c} D^{\alpha_{1}}$ to both sides of (29), we arrive at

$$
{ }^{c} D_{0}^{\alpha_{2}}\left(\left|{ }^{c} D_{0}^{\alpha_{1}} y(x)\right|^{p-2}{ }^{c} D_{0}^{\alpha_{1}} y(x)\right)=f(x, y(x)) .
$$

Therefore, $y$ is a solution of (1) on $[0, T]$. Summing up, we complete the proof of Proposition 10.

Since $T$ can be chosen arbitrarily large in Proposition 10, according to Definition 6, we have the following result.

Corollary 11. Let $y \in C([0,+\infty))$; then $y$ is a solution of problem (1) if and only if it satisfies the integral equation (19) on $[0,+\infty)$.

By Corollary 11, we obtain the following two existence results.

Remark 12. If $b_{0}=b_{1}=0$, and $f(x, 0)=0$ for $x \in(0,+\infty)$, then the constant function $y=0$ is a solution of problem (1).

Remark 13. If $f(x, y)=f(x)$ for $(x, y) \in(0,+\infty) \times \mathbb{R}$, then problem (1) has a solution on $[0,+\infty)$.

The following fixed point lemma is the main tool in the proofs of our results.

Lemma 14 (Schauder fixed point theorem). Let $\Omega$ be a closed, convex, and nonempty subset of a Banach space $Y$, and let $S$ : $\Omega \rightarrow \Omega$ be a mapping such that $\{S y: y \in \Omega\}$ is a relatively compact subset in $Y$. Then $S$ has at least one fixed point in $\Omega$.

\section{Main Results}

In this section, we will give and prove our main results in this paper.

Theorem 15. For any fixed initial values $b_{0}$ and $b_{1}$, there exists a sufficiently small constant $T^{*}>0$ such that problem (1) has a solution on $\left[0, T^{*}\right]$.

Proof. For any given positive constant $K$, choose $T^{*}>0$ sufficiently small which will be determined later. Let

$$
\Omega_{1}=\left\{y \in C\left(\left[0, T^{*}\right]\right) ; \sup _{0 \leq x \leq T^{*}}\left|y(x)-b_{0}\right| \leq K\right\} .
$$

Obviously, $\Omega_{1}$ is a closed, convex, and nonempty subset of $C\left(\left[0, T^{*}\right]\right)$. On this set $\Omega_{1}$ we define the operator $S$ :

$$
S y(x):=b_{0}+\frac{1}{\Gamma\left(\alpha_{1}\right)} \int_{0}^{x}(x-t)^{\alpha_{1}-1} \Phi_{y}(t) d t,
$$


where

$$
\begin{aligned}
& \Phi_{y}(x)=\phi_{q}\left(\phi_{p}\left(b_{1}\right)\right. \\
& \left.\quad+\frac{1}{\Gamma\left(\alpha_{2}\right)} \int_{0}^{x}(x-t)^{\alpha_{2}-1} f(t, y(t)) d t\right) .
\end{aligned}
$$

According to Proposition 10, in what follows, it suffices to show that the operator $S$ has a fixed point in $\Omega_{1}$.

Firstly, we will show that $S y \in \Omega_{1}$ for any $y \in \Omega_{1}$. To this end we begin by noting that for any $y \in \Omega_{1}, \Phi_{y} \in$ $C\left(\left[0, T^{*}\right]\right)$ by Remark 9 . Then we obtain that $S y \in C\left(\left[0, T^{*}\right]\right)$. Furthermore, for $x \in\left[0, T^{*}\right]$ we have

$$
\begin{aligned}
& \left|A y(x)-b_{0}\right|=\left|\frac{1}{\Gamma\left(\alpha_{1}\right)} \int_{0}^{x}(x-t)^{\alpha_{1}-1} \Phi_{y}(t) d t\right| \\
& \leq \frac{1}{\Gamma\left(\alpha_{1}\right)} \int_{0}^{x}(x-t)^{\alpha_{1}-1} \\
& \cdot \phi_{q}\left(\left|b_{1}\right|^{p-1}+\frac{M_{T^{*}, K} \Gamma(1-\sigma)}{\Gamma\left(\alpha_{2}-\sigma+1\right)} t^{\alpha_{2}-\sigma}\right) d t \\
& \leq \frac{T^{* \alpha_{1}}}{\Gamma\left(1+\alpha_{1}\right)}\left(\left|b_{1}\right|^{p-1}+\frac{M_{T^{*}, K} \Gamma(1-\sigma)}{\Gamma\left(\alpha_{2}-\sigma+1\right)}\right. \\
& \left.\cdot T^{* \alpha_{2}-\sigma}\right)^{q-1},
\end{aligned}
$$

where

$$
M_{T^{*}, K}=\sup _{x \in\left[0, T^{*}\right], y \in\left[b_{0}-K, b_{0}+K\right]} x^{\sigma}|f(x, y)| .
$$

Now we can choose $T^{*}>0$ so small that

$$
\begin{aligned}
& \frac{T^{* \alpha_{1}}}{\Gamma\left(1+\alpha_{1}\right)}\left(\left|b_{1}\right|^{p-1}+\frac{M_{T^{*}, K} \Gamma(1-\sigma)}{\Gamma\left(\alpha_{2}-\sigma+1\right)} T^{* \alpha_{2}-\sigma}\right)^{q-1} \\
& \quad \leq K
\end{aligned}
$$

which means that $S y \in \Omega_{1}$; that is, $S$ maps the set $\Omega_{1}$ to itself.

Secondly, we will also show that the family of functions $S \Omega_{1}=\left\{S y: y \in \Omega_{1}\right\}$ is a relatively compact set. That is to say, we need to show that $S \Omega_{1}$ is uniformly bounded and equicontinuous on $\left[0, T^{*}\right]$. The uniform boundedness follows from the definition of $\Omega_{1}$. As for the equicontinuity, for $0 \leq x_{1} \leq x_{2} \leq T^{*}$ and $y \in \Omega_{1}$, we see that

$$
\begin{gathered}
\left|A y\left(x_{1}\right)-A y\left(x_{2}\right)\right|=\frac{1}{\Gamma\left(\alpha_{1}\right)} \mid \int_{0}^{x_{1}}\left(x_{1}-t\right)^{\alpha_{1}-1} \\
\cdot \Phi_{y}(t) d t-\int_{0}^{x_{2}}\left(x_{2}-t\right)^{\alpha_{1}-1} \Phi_{y}(t) d t \mid \\
\leq \frac{1}{\Gamma\left(\alpha_{1}\right)}\left(\int_{0}^{x_{1}} \mid\left(\left(x_{1}-t\right)^{\alpha_{1}-1}-\left(x_{2}-t\right)^{\alpha_{1}-1}\right)\right. \\
\left.. \Phi_{y}(t)\left|d t+\int_{x_{1}}^{x_{2}}\left(x_{2}-t\right)^{\alpha_{1}-1}\right| \Phi_{y}(t) \mid d t\right)
\end{gathered}
$$

$$
\begin{aligned}
& \leq \frac{C}{\Gamma\left(\alpha_{1}\right)}\left(\int_{0}^{x_{1}}\left|\left(x_{1}-t\right)^{\alpha_{1}-1}-\left(x_{2}-t\right)^{\alpha_{1}-1}\right| d t\right. \\
& \left.+\int_{x_{1}}^{x_{2}}\left(x_{2}-t\right)^{\alpha_{1}-1} d t\right) \leq \frac{2 C}{\Gamma\left(\alpha_{1}+1\right)}\left(x_{2}-x_{1}\right)^{\alpha_{1}},
\end{aligned}
$$

where $C>0$ is independent of $x_{1}, x_{2}$, and $y$. Therefore $S \Omega_{1}$ is uniformly bounded and equicontinuous on $\left[0, T^{*}\right]$, and thus $S \Omega_{1}$ is a relatively compact subset of $C\left(\left[0, T^{*}\right]\right)$. By Lemma 14 there exists $y \in \Omega_{1}$ such that $S y=y$ and $y$ is a solution of problem (1) on $\left[0, T^{*}\right]$. The proof is completed.

We have proved the local existence of solutions of problem (1) in Theorem 15. However, maximal intervals of existence of those solutions are not necessarily $[0,+\infty)$, which change according to initial values $b_{0}$ and $b_{1}$. We give an example to illustrate it.

Example 16. Consider the following initial value problem for second-order ordinary differential equation

$$
\begin{aligned}
y^{\prime \prime}(x) & =2 y^{3}, \quad x>0, \\
y^{\prime}(0) & =\frac{1}{c^{2}}, \\
y(0) & =\frac{1}{c},
\end{aligned}
$$

where $c \neq 0$ is a constant. When $c<0$, it is easy to see that the function

$$
y(x)=\frac{1}{c-x}
$$

is a solution of $(38)$ on $[0,+\infty)$ and $[0,+\infty)$ is the maximum interval of existence. When $c>0$, however, the function $y$ is a solution of $(38)$ on $[0, c)$ and $[0, c)$ is the maximum interval of existence.

Under certain growth condition on the nonlinearity $f$ in (1), we will show that any solutions obtained in Theorem 15 can be extended to the interval $[0,+\infty)$ as solutions of problem (1).

Theorem 17. Suppose that there exist constant $\mu \in[0, p-1)$ and two nonnegative continuous functions $c_{1}(x), c_{2}(x)$ defined on $[0,+\infty)$ such that

$$
\begin{array}{r}
|f(x, y)| \leq \frac{1}{x^{\sigma}}\left(c_{1}(x)+c_{2}(x)|y|^{\mu}\right), \\
\quad(x, y) \in(0,+\infty) \times \mathbb{R} .
\end{array}
$$

Then maximum intervals of existence of any solutions of (1) obtained in Theorem 15 are $[0,+\infty)$.

Proof. Let $y_{T}$ be a solution of (1) on $[0, T]$ where $T>0$. By Definition 6 , it suffices to prove that for any fixed $\rho>0$, there exists a function $\tilde{y}$ as a solution of (1) on $[0, T+\rho]$ such that $\tilde{y}(x)=y_{T}(x)$ for $0 \leq x \leq T$. The proof will be completed by 
6

Advances in Mathematical Physics

applying Lemma 14 . Choose $K>0$ which will be determined later and denote

$$
\Omega_{2}=\left\{y \in C([T, T+\rho]) ; \sup _{T \leq x \leq T+\rho}|y(x)| \leq K\right\} .
$$

Obviously, $\Omega_{2}$ is a closed, convex, and nonempty subset of $C([T, T+\rho])$. On this set $\Omega_{2}$ we define the operator $S_{2}$ :

$$
\begin{array}{r}
S_{2} y(x):=z(x)+\frac{1}{\Gamma\left(\alpha_{1}\right)} \int_{T}^{x}(x-t)^{\alpha_{1}-1} \widetilde{\Phi}_{y}(t) d t, \\
T \leq x \leq T+\rho,
\end{array}
$$

where

$$
\begin{aligned}
& z(x)=b_{0}+\frac{1}{\Gamma\left(\alpha_{1}\right)} \int_{0}^{T}(x-t)^{\alpha_{1}-1} \Phi_{y_{T}}(t) d t \\
& \Phi_{y_{T}}(t)=\phi_{q}\left(\phi_{p}\left(b_{1}\right)\right. \\
& \left.+\frac{1}{\Gamma\left(\alpha_{2}\right)} \int_{0}^{t}(t-s)^{\alpha_{2}-1} f\left(s, y_{T}(s)\right) d s\right) \\
& \widetilde{\Phi}_{y}(t)=\phi_{q}\left(\phi_{p}\left(b_{1}\right)\right. \\
& \quad+\frac{1}{\Gamma\left(\alpha_{2}\right)} \int_{0}^{T}(t-s)^{\alpha_{2}-1} f\left(s, y_{T}(s)\right) d s \\
& \left.\quad+\frac{1}{\Gamma\left(\alpha_{2}\right)} \int_{T}^{t}(t-s)^{\alpha_{2}-1} f(s, y(s)) d s\right) .
\end{aligned}
$$

It is easy to see that $S_{2} y \in C([T, T+\rho])$ for any $y \in \Omega_{2}$. Further, by virtue of (40) we have

$$
\begin{aligned}
& \left|S_{2} y(x)\right| \leq|z(x)|+\left|\frac{1}{\Gamma\left(\alpha_{1}\right)} \int_{0}^{x}(x-t)^{\alpha_{1}-1} \widetilde{\Phi}_{y}(t) d t\right| \\
& \leq|z(x)|+\frac{1}{\Gamma\left(\alpha_{1}\right)} \int_{0}^{x}(x-t)^{\alpha_{1}-1} \phi_{q}\left(M_{0}+\frac{1}{\Gamma\left(\alpha_{2}\right)}\right. \\
& \left.\cdot \int_{T}^{t}(t-s)^{\alpha_{2}-1}|f(s, y(s))| d s\right) d t \leq|z(x)| \\
& +\frac{1}{\Gamma\left(\alpha_{1}\right)} \int_{0}^{x}(x-t)^{\alpha_{1}-1} \phi_{q}\left(M_{0}+\frac{1}{\Gamma\left(\alpha_{2}\right)}\right. \\
& \left.\cdot \int_{T}^{t}(t-s)^{\alpha_{2}-1} s^{-\sigma}\left(c_{1}(s)+K^{\mu} c_{2}(s)\right) d s\right) d t \\
& \leq|z(x)|+\frac{(T+\rho)^{\alpha_{1}}}{\Gamma\left(\alpha_{1}+1\right)} \phi_{q}\left(M_{0}+\frac{1}{\Gamma\left(\alpha_{2}\right)}\right. \\
& \left.\cdot \int_{T}^{T+\rho}(x-t)^{\alpha_{2}-1} t^{-\sigma}\left(c_{1}(t)+K^{\mu} c_{2}(t)\right) d t\right)
\end{aligned}
$$

$$
\begin{aligned}
& \leq \sup _{T \leq x \leq T+\rho}|z(x)|+\frac{(T+\rho)^{\alpha_{1}}}{\Gamma\left(\alpha_{1}+1\right)}\left(M_{0}+M_{1}\right. \\
& \left.+M_{2} K^{\mu}\right)^{q-1},
\end{aligned}
$$

where

$$
\begin{aligned}
& M_{0}=\left|b_{1}\right|^{p-1}+\frac{1}{\Gamma\left(\alpha_{2}\right)} \int_{0}^{T}(T-s)^{\alpha_{2}-1}\left|f\left(s, y_{T}\right)\right| d s, \\
& M_{1}=\frac{1}{\Gamma\left(\alpha_{2}\right)} \int_{T}^{T+\rho}(x-t)^{\alpha_{2}-1} t^{-\sigma} c_{1}(t) d t \\
& M_{2}=\frac{1}{\Gamma\left(\alpha_{2}\right)} \int_{T}^{T+\rho}(x-t)^{\alpha_{2}-1} t^{-\sigma} c_{2}(t) d t .
\end{aligned}
$$

Since $0 \leq \mu<p-1$, that is, $0 \leq \mu(q-1)<1$, we can choose $K$ so large that

$$
\left|S_{2} y(x)\right| \leq K, \quad x \in[T, T+\rho] .
$$

Then $S_{2}$ maps the set $\Omega_{2}$ to itself. To apply Lemma 14, we also need to show that the family of functions $S_{2} \Omega_{2}=\left\{S_{2} y\right.$ : $\left.y \in \Omega_{2}\right\}$ is a relatively compact set. That is to say, we need to show that $S_{2} \Omega_{2}$ is uniformly bounded and equicontinuous on $[T, T+\rho]$. The uniform boundedness follows from the definition of $\Omega_{2}$. As for the equicontinuity, we see that, for $T \leq x_{1} \leq x_{2} \leq T+\rho$ and $y \in \Omega_{2}$,

$$
\begin{aligned}
& \left|S_{2} y\left(x_{1}\right)-S_{2} y\left(x_{2}\right)\right| \leq\left|z\left(x_{1}\right)-z\left(x_{2}\right)\right| \\
& +\frac{1}{\Gamma\left(\alpha_{1}\right)} \mid \int_{T}^{x_{1}}\left(x_{1}-t\right)^{\alpha_{1}-1} \widetilde{\Phi}_{y}(t) d t-\int_{T}^{x_{2}}\left(x_{2}\right. \\
& -t)^{\alpha_{1}-1} \widetilde{\Phi}_{y}(t) d t \mid \\
& \quad \leq \frac{1}{\Gamma\left(\alpha_{1}\right)}\left(\int_{0}^{T} \mid\left(\left(x_{1}-t\right)^{\alpha_{1}-1}-\left(x_{2}-t\right)^{\alpha_{1}-1}\right)\right. \\
& +\Phi_{y_{T}}(t) \mid d t \\
& +\int_{T}^{x_{1}}\left|\left(\left(x_{1}-t\right)^{\alpha_{1}-1}-\left(x_{2}-t\right)^{\alpha_{1}-1}\right) \widetilde{\Phi}_{y}(t)\right| d t \\
& \left.\quad+\left|\int_{x_{1}}^{x_{2}}\left(x_{2}-t\right)^{\alpha_{1}-1} \widetilde{\Phi}_{y}(t) d t\right|\right) \\
& \quad \leq \frac{C}{\Gamma\left(\alpha_{1}\right)}\left(\int_{0}^{T}\left|\left(x_{1}-t\right)^{\alpha_{1}-1}-\left(x_{2}-t\right)^{\alpha_{1}-1}\right| d t\right. \\
& +\int_{T}^{x_{1}}\left|\left(x_{1}-t\right)^{\alpha_{1}-1}-\left(x_{2}-t\right)^{\alpha_{1}-1}\right| d t+\int_{x_{1}}^{x_{2}}\left(x_{2}\right. \\
& \left.\quad-t)^{\alpha_{1}-1} d t\right) \leq \frac{3 C}{\Gamma\left(\alpha_{1}+1\right)}\left(x_{1}-x_{2}\right)^{\alpha_{1}},
\end{aligned}
$$

where $C>0$ is independent of $x_{1}, x_{2}$, and $y$. Therefore $S_{2} \Omega_{2}$ is uniformly bounded and equicontinuous on $[T, T+\rho]$, and 
thus $S_{2} \Omega_{2}$ is a relatively compact subset in $C([T, T+\rho])$. By Lemma 14 there exists $y_{T+\rho} \in \Omega_{2}$ such that $S_{2} y_{T+\rho}=y_{T+\rho}$. Noting that $y_{T}(T)=y_{T+\rho}(T)$, we define

$$
\tilde{y}(x)= \begin{cases}y_{T}(x), & x \in[0, T], \\ y_{T+\rho}(x), & x \in(T, T+\rho] .\end{cases}
$$

From the definition of $S_{2}$, we see that $\tilde{y} \in C([0, T+\rho])$ is a solution of (1) on $[0, T+\rho]$, which completes the proof.

In a special case, an estimate of increasing rate for solutions as $x \rightarrow+\infty$ is made.

Corollary 18. Suppose that $x^{\sigma} f(x, y)$ is bounded on $[0,+\infty) \times \mathbb{R}$. Let $y$ be a solution of (1) on $[0,+\infty)$; then $y(x)=O\left(x^{\alpha_{1}+\left(\alpha_{2}-\sigma\right) /(p-1)}\right)(x \rightarrow+\infty)$.

Proof. According to Corollary 11, we have

$$
y(x)=b_{0}+\frac{1}{\Gamma\left(\alpha_{1}\right)} \int_{0}^{x}(x-t)^{\alpha_{1}-1} \Phi_{y}(t) d t .
$$

Note that

$$
\begin{aligned}
& \Phi_{y}(x)=\phi_{q}\left(\phi_{p}\left(b_{1}\right)\right. \\
& \left.+\frac{1}{\Gamma\left(\alpha_{2}\right)} \int_{0}^{x}(x-t)^{\alpha_{2}-1} f(t, y(t)) d t\right) \leq\left(\left|b_{1}\right|^{p-1}\right. \\
& \left.+\frac{1}{\Gamma\left(\alpha_{2}\right)} \int_{0}^{x}(x-t)^{\alpha_{2}-1}|f(t, y(t))| d t\right)^{q-1} \\
& \quad \leq\left(\left|b_{1}\right|^{p-1}+\frac{M}{\Gamma\left(\alpha_{2}\right)} \int_{0}^{x}(x-t)^{\alpha_{2}-1} t^{-\sigma} d t\right)^{q-1} \\
& \quad=\left(\left|b_{1}\right|^{p-1}+\frac{M \Gamma(1-\sigma)}{\Gamma\left(\alpha_{2}-\sigma+1\right)} x^{\alpha_{2}-\sigma}\right)^{q-1} \leq q\left|b_{1}\right| \\
& +q\left(\frac{M \Gamma(1-\sigma)}{\Gamma\left(\alpha_{2}-\sigma+1\right)}\right)^{q-1} x^{\left(\alpha_{2}-\sigma\right) /(p-1)},
\end{aligned}
$$

and we obtain

$$
\begin{aligned}
y(x) & \leq\left|b_{0}\right|+\frac{1}{\Gamma\left(\alpha_{1}\right)} \int_{0}^{x}(x-t)^{\alpha_{1}-1}\left|\Phi_{y}(t)\right| d t \\
& =c_{1}+c_{2} x^{\alpha_{1}}+c_{3} x^{\alpha_{1}+\left(\alpha_{2}-\sigma\right) /(p-1)},
\end{aligned}
$$

where $c_{1}, c_{2}, c_{3}>0$ is independent of $x$ and $y$. Since $0 \leq \sigma<$ $\alpha_{2}$, we conclude that

$$
y(x)=O\left(x^{\alpha_{1}+\left(\alpha_{2}-\sigma\right) /(p-1)}\right) \quad(x \longrightarrow+\infty) .
$$

\section{Example}

To illustrate our main results, we will present three examples.
Example 19. Consider the initial value problem for sequential fractional differential equation

$$
\begin{aligned}
{ }^{c} D_{0}^{0.1 c} D_{0}^{0.5} y(x) & =\frac{x+|y|^{\lambda}}{(1+x)(1+|y|)}, \quad x>0, \\
y(0) & =1, \\
{ }^{c} D_{0}^{0.5} y(0) & =1,
\end{aligned}
$$

where $\lambda \in(0,2)$. Here $p=2, \alpha_{1}=0.5, \alpha_{2}=0.1$, and $f(x, y)=$ $\left(x+|y|^{\lambda}\right) /(1+x)(1+|y|)$. It is easy to see that

$$
|f(x, y)| \leq \begin{cases}\frac{x}{1+x}+\frac{|y|^{\lambda}}{1+x}, & \lambda \in(0,1), \\ \frac{x}{1+x}+\frac{|y|^{\lambda-1}}{1+x}, & \lambda \in(1,2), \\ \frac{x}{1+x}+\frac{|y|^{0.5}}{1+x}, & \lambda=1,\end{cases}
$$

for $(x, y) \in[0, \infty) \times \mathbb{R}$. According to Theorem 17 , problem (53) has at least one positive solution on $[0,+\infty)$ for any fixed $\lambda \in(0,2)$. Let $y_{\lambda}$ be a positive solution of $(53)$ on $[0,+\infty)$. Since $|f(x, y)|$ is bounded on $[0,+\infty) \times \mathbb{R}$ when $\lambda \in(0,1]$, we further have an estimate of increasing rate for $y_{\lambda}$ by Corollary 18:

$$
y_{\lambda}(x)=O\left(x^{0.6}\right) \quad(x \longrightarrow+\infty) .
$$

Example 20. Consider the initial value problem for nonlinear fractional differential equation

$$
\begin{aligned}
& { }^{c} D_{0}^{0.75}\left(\left|{ }^{c} D_{0}^{0.5} y(x)\right|^{p-2}{ }^{c} D_{0}^{0.5} y(x)\right) \\
& =x^{-0.5}+x^{-0.25} \sin y, \quad x>0, \\
& y(0)=1, \\
& { }^{c} D_{0}^{0.5} y(0)=0,
\end{aligned}
$$

where $p>1$. Here $\alpha_{1}=0.5, \alpha_{2}=0.75$, and $f(x, y)=x^{-0.5}+$ $x^{-0.25} \sin y$. Choosing $\sigma=0.5 \in[0,0.75)$, we see that

$$
\begin{aligned}
x^{\sigma}|f(x, y)| & =x^{0.5}\left|x^{-0.5}+x^{-0.25} \sin y\right| \\
& \leq 1+x^{0.25}|y|^{\mu}
\end{aligned}
$$

for $(x, y) \in(0, \infty) \times \mathbb{R}$, where $\mu \in(0, p-1)$. According to Theorem 17, problem (56) has at least one solution on $[0,+\infty)$. Let $y$ be a solution of $(56)$ on $[0,+\infty)$. By simple computation similar to that in the proof of Corollary 18, we further have an estimate of increasing rate for $y$ :

$$
y(x)=O\left(x^{p / 2(p-1)}\right) \quad(x \longrightarrow+\infty) .
$$


Example 21. Consider the initial value problems for ordinary differential equations with $p$-Laplacian

$$
\begin{aligned}
\left(\left|y^{\prime}(x)\right|^{p-2} y^{\prime}(x)\right)^{\prime} & =\ln x+x^{-0.5} \arctan y, \quad x>0, \\
y(0) & =1, \\
{ }^{c} D_{0}^{0.5} y(0) & =0,
\end{aligned}
$$

where $p>2$. Here $\alpha_{1}=\alpha_{2}=1$ and $f(x, y)=\ln x+$ $x^{-0.5} \arctan y$. Choosing $\sigma=0.5 \in[0,0.75)$, we see that

$$
\begin{aligned}
x^{\sigma}|f(x, y)| & =x^{0.5}\left|\ln x+x^{-0.5} \arctan y\right| \\
& \leq x^{0.5}|\ln x|+|y|
\end{aligned}
$$

for $(x, y) \in(0, \infty) \times \mathbb{R}$. According to Theorem 17, problem (59) has at least one solution on $[0,+\infty)$. Let $y$ be a solution of $(59)$ on $[0,+\infty)$. By simple computation similar to that in the proof of Corollary 18, we further have an estimate of increasing rate for $y$ :

$$
y(x)=O\left(x^{(p+1) /(p-1)}\right) \quad(x \longrightarrow+\infty) .
$$

\section{Conflict of Interests}

The authors declare that there is no conflict of interests regarding the publication of this paper.

\section{Acknowledgments}

The second author was supported in part by National Natural Science Foundation of China (no. 11071099), CPSF (no. 2015M572301), and the Fundamental Research Funds for the Central Universities (no. 2015ZM191).

\section{References}

[1] I. Podlubny, Fractional Differential Equations, Academic Press, New York, NY, USA, 1999.

[2] K. S. Miller and B. Ross, An Introduction to the Fractional Calculus and Differential Equations, John Wiley \& Sons, New York, NY, USA, 1993.

[3] A. A. Kilbas, H. M. Srivastava, and J. J. Trujillo, Theory and Applications of Fractional Differential Equations, Elsevier Science B.V., Amsterdam, The Netherlands, 2006.

[4] K. Diethelm, The Analysis of Fractional Differential Equations, Springer, Berlin, Germany, 2010.

[5] R. P. Agarwal, M. Benchohra, and S. Hamani, "A survey on existence results for boundary value problems of nonlinear fractional differential equations and inclusions," Acta Applicandae Mathematicae, vol. 109, no. 3, pp. 973-1033, 2010.

[6] Z. Zhang, F. Zeng, and G. E. Karniadakis, "Optimal error estimates of spectral petrov-galerkin and collocation methods for initial value problems of fractional differential equations," SIAM Journal on Numerical Analysis, vol. 53, no. 4, pp. 20742096, 2015.

[7] J. Čermák and T. Kisela, "Stability properties of two-term fractional differential equations," Nonlinear Dynamics, vol. 80, no. 4, pp. 1673-1684, 2015.
[8] T. Jankowski, "Systems of nonlinear fractional differential equations," Fractional Calculus and Applied Analysis, vol. 18, no. 1, pp. 122-132, 2015.

[9] K. Diethelm and A. D. Freed, "On the soluton of nonlinear fractional order differential equations used in the modeling of viscoplasticity," in Scientific Computing in Chemical Engineering II. Computational Flluid Dynamics, Reaction Engineering and Molecular Porperties, F. Keil, W. Mackens, H. Voss, and J. Werther, Eds., pp. 217-224, Springer, Heidelberg, Germany, 1999.

[10] C. Giannantoni, "The problem of the initial conditions and their physical meaning in linear differential equations of fractional order," Applied Mathematics and Computation, vol. 141, no. 1, pp. 87-102, 2003.

[11] R. Hilfer, Applications of Fractional Calculus in Physics, World Scientific, Singapore, 2000.

[12] N. Laskin, "Fractional Schrödinger equation," Physical Review E, vol. 66, no. 5, Article ID 056108, pp. 1-7, 2002.

[13] D. Băleanu, O. G. Mustafa, and R. P. Agarwal, "On the solution set for a class of sequential fractional differential equations," Journal of Physics. A. Mathematical and Theoretical, vol. 43, no. 38, Article ID 385209, 2010.

[14] D. Băleanu, O. G. Mustafa, and R. P. Agarwal, "On $L^{p}$-solutions for a class of sequential fractional differential equations," Applied Mathematics and Computation, vol. 218, no. 5, pp. 20742081, 2011.

[15] Q. Li, H. Su, and Z. Wei, "Existence and uniqueness result for a class of sequential fractional differential equations," Journal of Applied Mathematics and Computing, vol. 38, no. 1-2, pp. 641652, 2012.

[16] Z. Wei, Q. Li, and J. Che, "Initial value problems for fractional differential equations involving Riemann-Liouville sequential fractional derivative," Journal of Mathematical Analysis and Applications, vol. 367, no. 1, pp. 260-272, 2010.

[17] C. Bai, "Impulsive periodic boundary value problems for fractional differential equation involving Riemann-Liouville sequential fractional derivative," Journal of Mathematical Analysis and Applications, vol. 384, no. 2, pp. 211-231, 2011.

[18] A. Alsaedi, S. K. Ntouyas, R. P. Agarwal, and B. Ahmad, "On Caputo type sequential fractional differential equations with nonlocal integral boundary conditions," Advances in Difference Equations, vol. 2015, article 33, 2015.

[19] B. Ahmad and S. K. Ntouyas, "Existence results for a coupled system of Caputo type sequential fractional differential equations with nonlocal integral boundary conditions," Applied Mathematics and Computation, vol. 266, pp. 615-622, 2015.

[20] B. Ahmad and J. J. Nieto, "Sequential fractional differential equations with three-point boundary conditions," Computers \& Mathematics with Applications, vol. 64, no. 10, pp. 3046-3052, 2012.

[21] Y. Chen and X. Tang, "Solvability of sequential fractional order multi-point boundary value problems at resonance," Applied Mathematics and Computation, vol. 218, no. 14, pp. 7638-7648, 2012.

[22] W. Jiang, "Solvability of fractional differential equations with $p$ Laplacian at resonance," Applied Mathematics and Computation, vol. 260, pp. 48-56, 2015.

[23] W. Ge and J. Ren, "An extension of Mawhin's continuation theorem and its application to boundary value problems with 
a p-Laplacain," Nonlinear Analysis, Theory, Methods and Applications, vol. 58, no. 3-4, pp. 477-488, 2004.

[24] H. Ye and R. Huang, "Initial value problem for nonlinear fractional differential equations with sequential fractional derivative," Advances in Difference Equations, vol. 2015, article 291, 2015. 


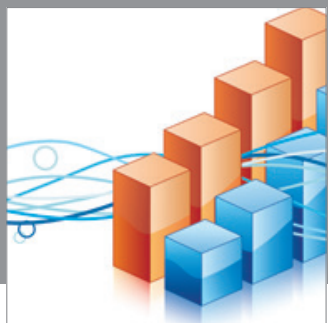

Advances in

Operations Research

mansans

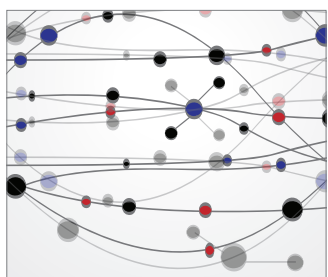

The Scientific World Journal
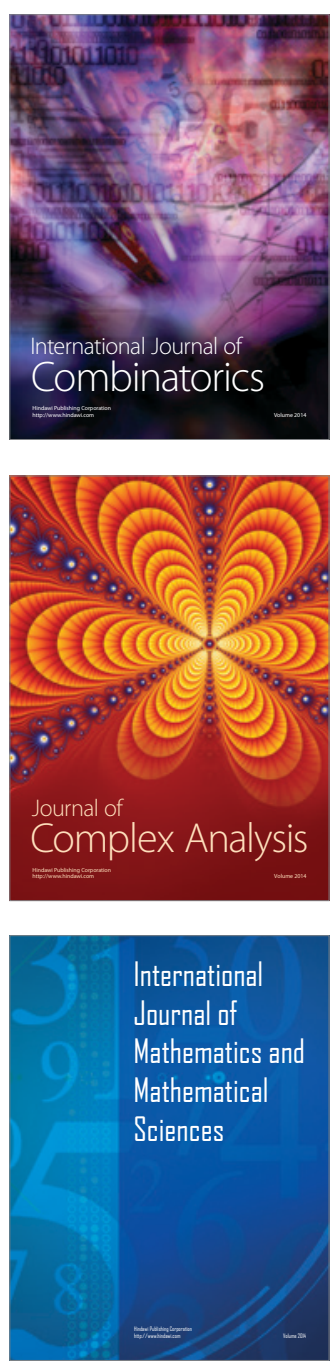
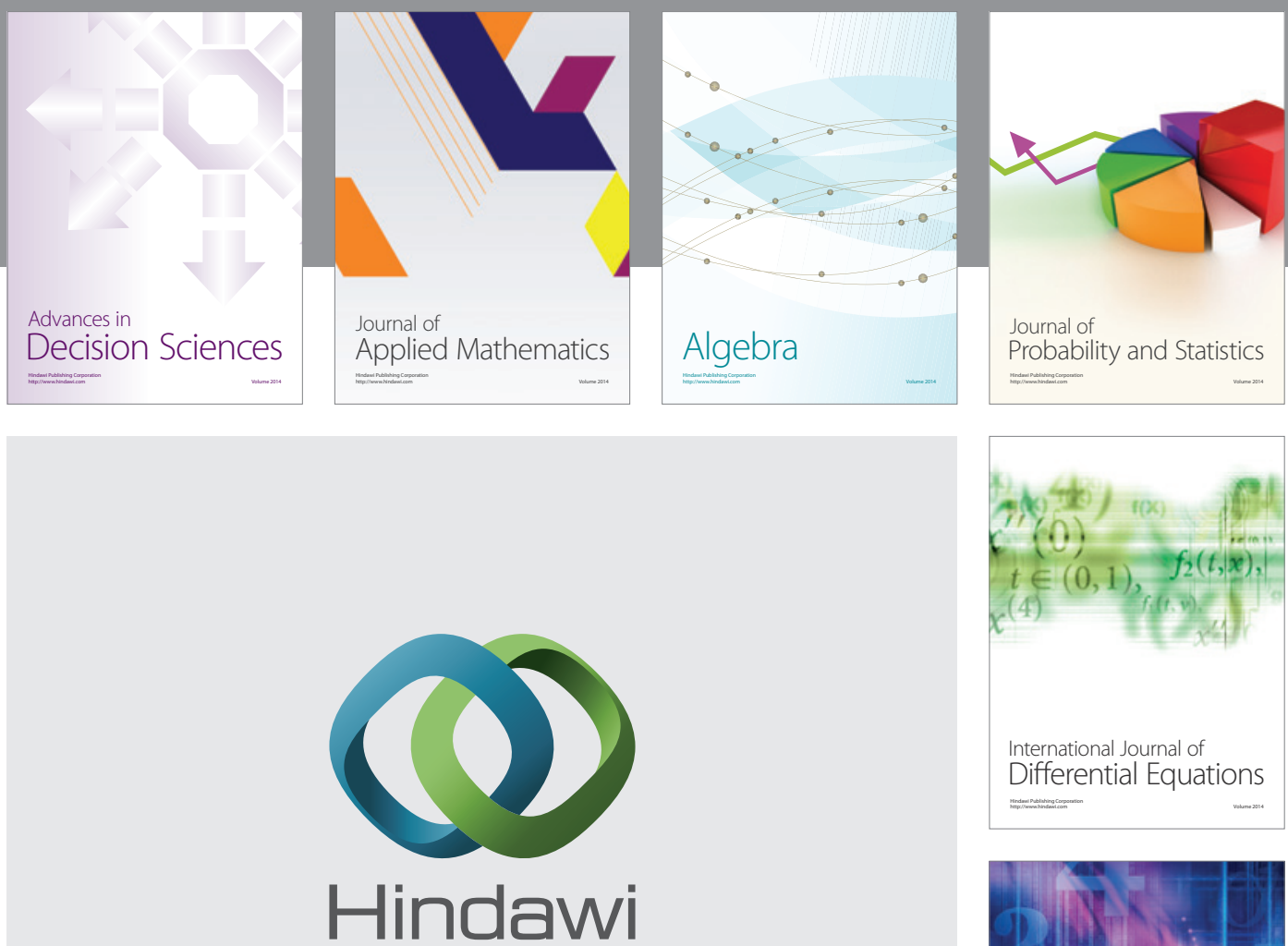

Submit your manuscripts at http://www.hindawi.com
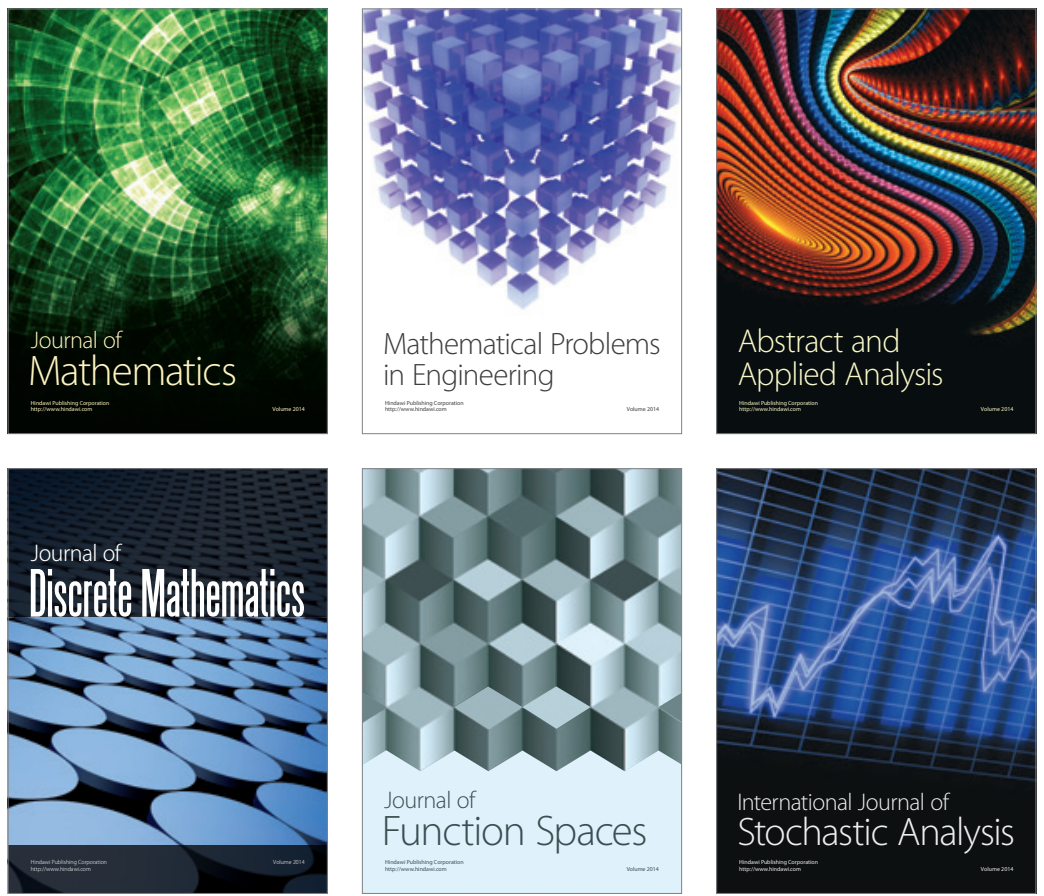

Journal of

Function Spaces

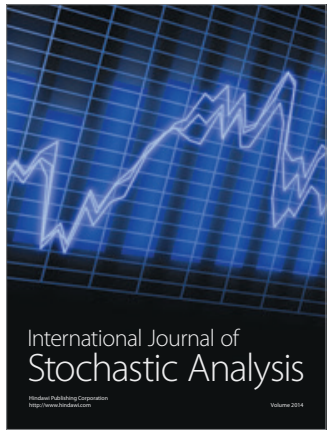


